\title{
SCIENTIFIC REPORTS

\section{Differentiation of multiple system atrophy from Parkinson's disease by structural connectivity derived from probabilistic tractography}

\author{
Alexandra Abos $\mathbb{1}^{1}$, Hugo C. Baggio ${ }^{1}$, Barbara Segura ${ }^{1,2}$, Anna Campabadal ${ }^{1}$, Carme Uribe ${ }^{1}{ }^{1}$ \\ Darly Milena Giraldo 2,3, Alexandra Perez-Soriano ${ }^{2,3}$, Esteban Muñoz ${ }^{2,3,4}$, Yaroslau Compta ${ }^{2,3,4}$, \\ Carme Junque ${ }^{1,2,4}$ \& Maria Jose Marti ${ }^{2,3,4^{*}}$
}

Recent studies combining diffusion tensor-derived metrics and machine learning have shown promising results in the discrimination of multiple system atrophy (MSA) and Parkinson's disease (PD) patients. This approach has not been tested using more complex methodologies such as probabilistic tractography. The aim of this work is assessing whether the strength of structural connectivity between subcortical structures, measured as the number of streamlines (NOS) derived from tractography, can be used to classify MSA and PD patients at the single-patient level. The classification performance of subcortical FA and MD was also evaluated to compare the discriminant ability between diffusion tensor-derived metrics and NOS. Using diffusion-weighted images acquired in a 3T MRI scanner and probabilistic tractography, we reconstructed the white matter tracts between 18 subcortical structures from a sample of 54 healthy controls, 31 MSA patients and 65 PD patients. NOS between subcortical structures were compared between groups and entered as features into a machine learning algorithm. Reduced NOS in MSA compared with controls and PD were found in connections between the putamen, pallidum, ventral diencephalon, thalamus, and cerebellum, in both right and left hemispheres. The classification procedure achieved an overall accuracy of $78 \%$, with $71 \%$ of the MSA subjects and $86 \%$ of the PD patients correctly classified. NOS features outperformed the discrimination performance obtained with FA and MD. Our findings suggest that structural connectivity derived from tractography has the potential to correctly distinguish between MSA and PD patients. Furthermore, NOS measures obtained from tractography might be more useful than diffusion tensor-derived metrics for the detection of MSA.

Multiple system atrophy (MSA) is a sporadic, progressive neurodegenerative disease characterized by autonomic failure, parkinsonian symptoms, and/or cerebellar features. Striatonigral degeneration and olivopontocerebellar atrophy are typically found at postmortem examination of MSA patients, reflecting the presence of parkinsonian features and ataxia, respectively ${ }^{1}$. Although MSA may resemble Parkinson's disease (PD) in its early stages, brain damage is more aggressive, with usually no response to dopaminergic medication, and leading to a rapidly progressive disease course with a fatal prognosis ${ }^{1,2}$. For this reason, improving our ability to diagnose and to predict MSA progression after diagnosis is a major objective in clinical practice.

Conventional diagnostic magnetic resonance imaging (MRI) has been widely used as a complementary tool in the differential diagnosis between PD and MSA. In most cases of PD, clinical MR imaging shows no abnormalities until advanced disease stages, and brain degeneration is typically not as extensive as in MSA ${ }^{3}$. Typical radiological features in MSA are mainly located in subcortical structures, including a cruciform hyperintensity in the pons, called the "hot cross bun sign"; changes in the putamen comprising atrophy and T2 signal hypointensity,

${ }^{1}$ Medical Psychology Unit, Department of Medicine, Institute of Neuroscience, University of Barcelona, Barcelona, Catalonia, Spain. ${ }^{2}$ Centro de Investigación Biomédica en Red sobre Enfermedades Neurodegenerativas (CIBERNED), Hospital Clínic de Barcelona, Barcelona, Catalonia, Spain. ${ }^{3}$ Movement Disorders Unit, Neurology Service, Hospital Clínic de Barcelona. Institute of Neuroscience,University of Barcelona, Barcelona, Catalonia, Spain. ${ }^{4}$ Institute of Biomedical Research August Pi i Sunyer (IDIBAPS), Barcelona, Catalonia, Spain. *email: MJMARTI@clinic.cat 


\begin{tabular}{|l|l|l|l|l|}
\hline & HC $(\mathbf{n}=\mathbf{5 4})$ & $\mathbf{P D}(\mathbf{n}=\mathbf{6 5})$ & MSA $(\mathbf{n}=\mathbf{3 1})$ & Stat $/ \mathbf{p}$ \\
\hline Age & $64.3(11.3)$ & $65.4(10)$ & $60.9(8.4)$ & $2.0434 / \mathrm{p}=0.3317$ \\
\hline Years of education & $12.63(4.3)$ & $12.48(5.4)$ & $10.68(3.8)$ & $1.9333 / \mathrm{p}=0.3803$ \\
\hline Sex (male/female) & $26 / 28$ & $48 / 17$ & $19 / 12$ & $4.2921 / \mathrm{p}=0.0436^{* 1}$ \\
\hline Years of evolution & - & $8.26(6.02)$ & $4.46(2.75)$ & $3.3466 / \mathrm{p}<0.001^{* 3}$ \\
\hline H\&Y (1:2:3:4:5) & - & $10: 32: 22: 1: 0$ & $0: 8: 11: 9: 3$ & $5.77 / \mathrm{p}<0.001^{* 3}$ \\
\hline UPDRS & - & $16.59(9.22)$ & - & - \\
\hline UMSARS & - & - & $49.97(20.46)$ & - \\
\hline LEDD & - & $639.35(382.76)$ & $520.67(426.4)$ & $1.347 / \mathrm{p}=0.0936$ \\
\hline
\end{tabular}

Table 1. Sociodemographic and clinical characteristics by group. HC: healthy controls; PD: Parkinson's disease patient group; MSA: multiple system atrophy patient group; H\&Y: Hoehn and Yahr scale; UPDRS: United Parkinson's Disease Rating Scale, motor section scores; UMSARS: United Multiple System Atrophy Rating Scale, motor section scores; LEDD: levodopa equivalent daily dose (in mg); * refers to significant results; Posthoc differences between $\mathrm{HC}$ and $\mathrm{PD}^{1} ; \mathrm{HC}$ and $\mathrm{MSA}^{2} ; \mathrm{PD}^{2}$ and $\mathrm{MSA}^{3}$.

with a marginal hyperintensity; and atrophy of the cerebellar peduncles (chiefly the middle cerebellar peduncle (MCP)), pons, and cerebellum ${ }^{4}$.

The use of 3 T MR scanning alongside advanced analysis techniques has led to improvements in the diagnostic value of MRI. Diffusion-weighted MRI (DWI), especially using diffusion tensor imaging (DTI), is one of the most common MRI techniques when studying neurodegenerative diseases, as it allows detecting microstructural abnormalities and assessing the integrity of white matter (WM) tracts. Previous DTI studies in MSA have characterized diffusion abnormalities with different approaches such as using regions of interest (ROI) and tract-based spatial statistics (TBSS). Disrupted WM integrity, decreased streamline density and abnormal diffusivity measures were observed in subcortical structures such as the basal ganglia, middle cerebellar peduncles, cerebellum, and pons ${ }^{5-8}$.

Tractography allows reconstructing brain WM pathways, which helps understand how the brain operates as a connected system. Furthermore, apart from quantifying the local streamline density, tractography can be used to reconstruct the structural connectome - i.e., a comprehensive description of the structural connections between brain regions ${ }^{9}$. In recent years, structural connectivity has been studied in PD patients, showing reduced structural connectivity between the substantia nigra and the striatum and thalamus in these patients ${ }^{10-12}$. Furthermore, reduced fiber density has been observed between the associative and limbic cortex, putamen, thalamus, caudate, and globus pallidus in PD compared with controls ${ }^{13}$. Given that PD is a very heterogeneous disease with both motor and non-motor symptoms, structural connectivity has also been used to study subgroups with different predominant symptomatology. Structural connectivity differences were observed in PD with and without tremor $^{14}$, freezing of gate ${ }^{15-17}, \mathrm{PD}-\mathrm{MCI}^{18}$, and different motor subtypes ${ }^{19}$. However, although studying the connectome has proven useful to detect structural abnormalities in PD, as far as we know, limited work has been done in terms of characterizing MSA connectivity pattern using tractography ${ }^{20,21}$.

With the introduction of machine learning algorithms, MRI studies have been able to test the importance of specific measures in discriminating different diseases or conditions. As DTI has been useful in characterizing subcortical abnormalities in MSA, diffusion measures such as fractional anisotropy (FA) and mean diffusivity (MD) have been used as features to differentiate between PD and MSA patients. Sensitivities and specificities around $80 \%$ have been achieved in most studies ${ }^{22-25}$. These results seem to indicate that diffusion tensor-derived metrics might be useful for discriminating between MSA and PD. FA and MD are commonly used to detect microstructural abnormalities in subcortical structures, but no information about the relationship between regions can be obtained from these measures. Tractography allows assessing whether the connectivity between these structures is also impaired, which is relevant to understand the pathological pathways of neurodegenerative diseases. Thus, tractography-derived metrics might be of interest to identify specific abnormal brain connections with higher discriminating power. To the best of our knowledge, no previous published works focused on combining structural connectivity and machine learning to discriminate PD from MSA patients.

In the present study, we use tractography to discriminate patients with MSA from patients with PD. Our hypothesis is that structural connectivity between subcortical structures is informative enough to distinguish MSA from PD at the individual-subject level. To test this hypothesis, we passed the connectivity data into a supervised machine learning algorithm and assessed its ability to correctly determine each patient's group membership. Additionally, we hypothesize that subcortical structural connectivity derived from tractography is more informative than previously studied diffusion tensor-derived metrics.

\section{Results}

Subjects. Table 1 shows the sociodemographic and clinical characteristics of the samples. No significant intergroup differences were observed for age and years of education $(\mathrm{p}=0.3317$ and $\mathrm{p}=0.3803$, respectively). A significant intergroup effect was observed for gender distribution $(\mathrm{p}=0.0436)$.

Comparing the two groups of patients, no significant differences were found between PD and MSA patients for age, years of education, gender, or levodopa equivalent daily dose (LEDD) $(p=0.0668, p=0.1234, p=0.3141$, and $\mathrm{p}=0.0936$ respectively). MSA patients differed from PD participants only in disease duration and Hoehn and Yahr scores $(\mathrm{H} \& \mathrm{Y})$ (both $\mathrm{p}<0.001)$. 


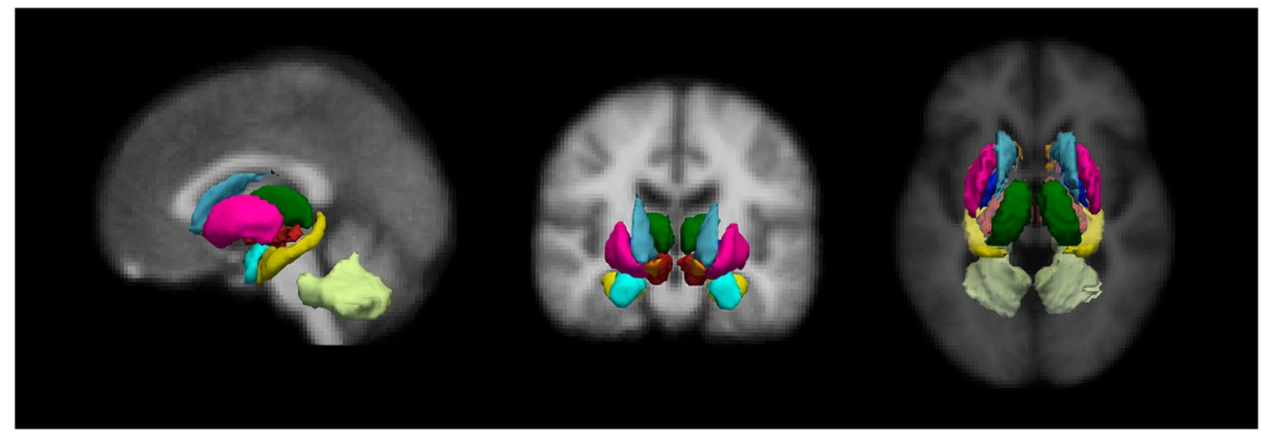

Figure 1. Eighteen subcortical region of interest (ROI) from FreeSurfer. Representation of the subcortical parcellation used in this study (for right and left hemispheres): the bilateral nucleus accumbens; amygdala; caudate nucleus; hippocampus; pallidum; putamen; thalamus; ventral diencephalon (including the hypothalamus, mammillary body, subthalamic nuclei, substantia nigra, red nucleus) and cerebellar white matter, including the middle cerebellar peduncles.

mean significant NOS

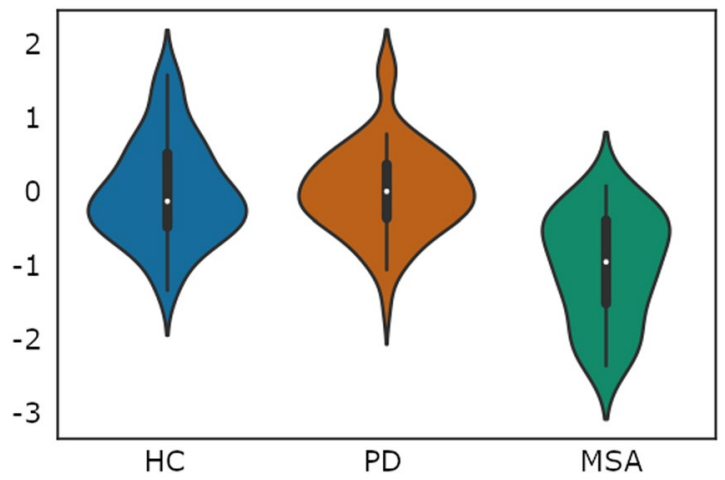

Figure 2. Plot illustrates the distribution of the average number of streamlines (NOS) between the 10 significantly reduced tracts found in MSA patients using threshold-free network based statistics (TFNBS). NOS values were Z-transformed to calculate the global mean value comprising all significant connections; HC: healthy controls; PD: Parkinson's disease group; MSA: multiple system atrophy group. Plot width represents the frequency (density) of values; the height indicates the upper (max) and lower (min) limits.

Although patients showed no significant demographical differences, these characteristics could influence and bias the performance of the classifier. We therefore repeated the classification algorithm using a better matched subsample of PD and MSA patients with equal sample sizes $(n=30$ in both groups $)$ and no significant differences in age, years of education, gender, or LEDD $(\mathrm{p}=0.1969, \mathrm{p}=0.7203, \mathrm{p}=0.8223$, and $\mathrm{p}=0.1692$ respectively) (Supplementary Table 1). Concretely, PD patient were randomly sampled based on the proportion of male/female patients and the demographic characteristics of MSA patients.

Structural connectivity analysis. The nodes used to construct the structural connectivity network were derived from the automated FreeSurfer segmentation ${ }^{26,27}$, which includes 18 subcortical structures (Fig. 1). The connectivity between regions was represented as the number of streamlines between them (NOS). Significant differences in NOS were found between groups in 10 subcortical connections (Fig. 2 and Table 2) using a method called threshold-free network based statistics (TFNBS) ${ }^{28}$, which performs statistical inference on brain graphs. TFNBS works similarly to network-based statistics (NBS) ${ }^{29}$ but without requiring the definition of a component-defining threshold and generating edge-wise significance values. The subcortical structures linked to connections showing reduced NOS in the MSA group compared with healthy controls (HC) and PD were the putamen, the pallidum, the ventral diencephalon, the thalamus, and the cerebellum, in both right and left hemispheres (Supplementary Fig. 1). Post-hoc testing showed reduced connectivity in all 10 connections in MSA patients compared with HC (Fig. 3A) and in eight connections in MSA compared with PD patients (Fig. 3B). No connections showed significantly higher NOS in PD or MSA patients compared with HC, or in MSA compared with PD subjects. In PD patients compared with $\mathrm{HC}$, only the connection between the putamen and the thalamus in the right hemisphere was found to be significantly reduced (Fig. 3C).

ROI analysis. Diffusion tensor-derived metrics (FA and MD) extracted from the 18 subcortical ROIs were evaluated as a measure of microstructural integrity. Significant FA differences surviving FDR correction were found in the bilateral ventral diencephalon, left amygdala, right thalamus, and right cerebellum. Significant 


\begin{tabular}{|l|l|l|l|l|}
\hline & HC $(\mathbf{n}=\mathbf{5 4})$ & PD $(\mathbf{n}=\mathbf{6 5})$ & MSA $(\mathbf{n}=\mathbf{3 1})$ & Stat $/ \mathbf{p}$ \\
\hline Left Pallidum-Left Putamen & $1.4874 \cdot 10^{6}\left(0.2522 \cdot 10^{6}\right)$ & $1.5197 \cdot 10^{6}\left(0.2627 \cdot 10^{6}\right)$ & $1.2184 \cdot 10^{6}\left(0.2599 \cdot 10^{6}\right)$ & $\mathrm{F}=5.1788 / \mathrm{p}<0.001^{2,3 *}$ \\
\hline Left Hippocampus-Left Thalamus & $1.023 \cdot 10^{6}\left(0.2549 \cdot 10^{6}\right)$ & $0.9295 \cdot 10^{6}\left(0.2975 \cdot 10^{6}\right)$ & $0.7847 \cdot 10^{6}\left(0.2147 \cdot 10^{6}\right)$ & $\mathrm{F}=2.4171 / \mathrm{p}=0.0092^{2,3 *}$ \\
\hline Left Pallidum-Left VentralDC & $1.1713 \cdot 10^{6}\left(0.4372 \cdot 10^{6}\right)$ & $1.2162 \cdot 10^{6}\left(0.4139 \cdot 10^{6}\right)$ & $0.8819 \cdot 10^{6}\left(0.3154 \cdot 10^{6}\right)$ & $\mathrm{F}=3.6096 / \mathrm{p}=0.0065^{2,3 *}$ \\
\hline Left VentralDC-Left Cerebellum & $0.7598 \cdot 10^{6}\left(0.2172 \cdot 10^{6}\right)$ & $0.8399 \cdot 10^{6}\left(0.2092 \cdot 10^{6}\right)$ & $0.5747 \cdot 10^{6}\left(0.2734 \cdot 10^{6}\right)$ & $\mathrm{F}=5.2403 / \mathrm{p}<0.001^{2,3 *}$ \\
\hline Right Pallidum-Right Putamen & $1.3556 \cdot 10^{6}\left(0.2265 \cdot 10^{6}\right)$ & $1.4148 \cdot 10^{6}\left(0.2195 \cdot 10^{6}\right)$ & $1.1536 \cdot 10^{6}\left(0.3044 \cdot 10^{6}\right)$ & $\mathrm{F}=4.7647 / \mathrm{p}<0.001^{2,3 *}$ \\
\hline Right Putamen-Right Thalamus & $0.7936 \cdot 10^{6}\left(0.2888 \cdot 10^{6}\right)$ & $0.6769 \cdot 10^{6}\left(0.2435 \cdot 10^{6}\right)$ & $0.5442 \cdot 10^{6}\left(0.3885 \cdot 10^{6}\right)$ & $\mathrm{F}=1.9587 / \mathrm{p}=0.0065^{1,2 *}$ \\
\hline Right Thalamus -Right VentralDC & $2.7867 \cdot 10^{6}\left(0.4611 \cdot 10^{6}\right)$ & $2.8886 \cdot 10^{6}\left(0.4822 \cdot 10^{6}\right.$ & $2.4535 \cdot 10^{6}\left(0.5392 \cdot 10^{6}\right)$ & $\mathrm{F}=3.8987 / \mathrm{p}=0.0042^{2,3 *}$ \\
\hline Left VentralDC-Right Cerebellum & $0.0918 \cdot 10^{6}\left(0.0623 \cdot 10^{6}\right)$ & $0.0834 \cdot 10^{6}\left(0.0503 \cdot 10^{6}\right)$ & $0.0471 \cdot 10^{6}\left(0.0426 \cdot 10^{6}\right)$ & $\mathrm{F}=3.1419 / \mathrm{p}=0.0096^{2,3 *}$ \\
\hline Left Cerebellum -Right Cerebellum & $4.4335 \cdot 10^{6}\left(1.4225 \cdot 10^{6}\right)$ & $4.1623 \cdot 10^{6}\left(1.2313 \cdot 10^{6}\right)$ & $2.3522 \cdot 10^{6}\left(1.032 \cdot 10^{6}\right)$ & $\mathrm{F}=6.7853 / \mathrm{p}<0.001^{2,3 *}$ \\
\hline Right VentralDC-Right Cerebellum & $0.8462 \cdot 10^{6}\left(0.2694 \cdot 10^{6}\right)$ & $0.9045 \cdot 10^{6}\left(0.2629 \cdot 10^{6}\right)$ & $0.5694 \cdot 10^{6}\left(0.2649 \cdot 10^{6}\right)$ & $\mathrm{F}=5.722 / \mathrm{p}<0.001^{2,3 *}$ \\
\hline
\end{tabular}

Table 2. Significant reduced connections found with TFNBS by group. HC: healthy controls; PD: Parkinson's disease patient group; MSA: multiple system atrophy patient group; * significant FDR corrected results; Posthoc differences between $\mathrm{HC}$ and $\mathrm{PD}^{1}$; $\mathrm{HC}$ and $\mathrm{MSA}^{2}$; $\mathrm{PD}$ and $\mathrm{MSA}^{3}$; Stats refers to F-test $(\mathrm{F})$.

A

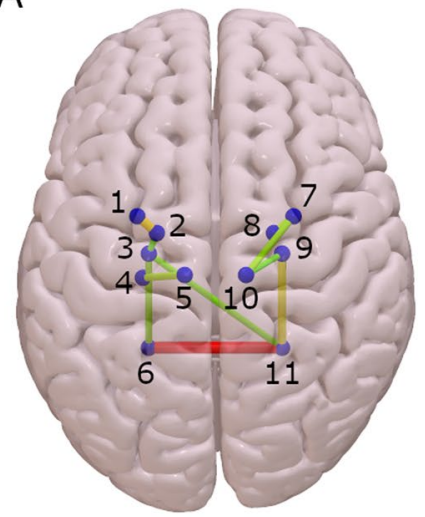

B

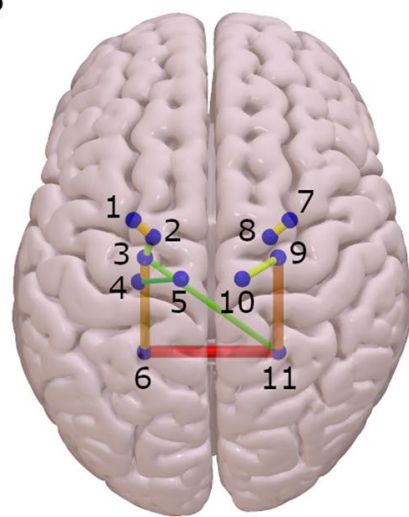

C

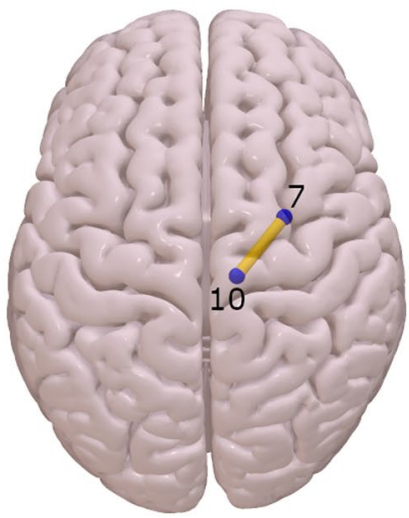

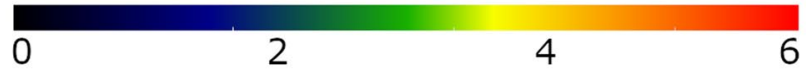

Figure 3. Connectivity differences between groups patients using threshold-free network based statistics (TFNBS). (A) Connectivity differences between healthy controls and multiple system atrophy patients. (B) Connectivity differences between Parkinson's disease and multiple system atrophy patients. (C) Connectivity differences between healthy controls and Parkinson's disease patients. Brain edges are scaled according to the value of the T statistic (shown in the color bar), $\mathrm{p}<0.05$, FDR corrected. 1: Left Putamen; 2: Left Pallidum; 3: Left Ventral diencephalon; 4: Left Hippocampus; 5: Left Thalamus; 6: Left Cerebellum; 7: Right Putamen; 8: Right Pallidum; 9: Right Ventral diencephalon; 10: Right Thalamus; 11: Right Cerebellum.

MD differences were detected in the nucleus accumbens, putamen and ventral diencephalon in the left hemisphere, and in the pallidum, thalamus and cerebellum in the right hemisphere. Post-hoc testing showed diffusion abnormalities (reduced FA and increased MD) in all structures in the MSA group compared with the HC and PD samples, except for the left nucleus accumbens, where no MD increments were found between MSA and PD. Abnormal FA and MD values in PD compared with HC were found in the thalamus and the putamen. Furthermore, increased FA was detected in MSA patients compared with HC and PD patients in the left amygdala. A summary of the significant FA and MD results is shown in Tables 3 and 4 . A detailed description is given in Supplementary Tables 2 and 3.

Intergroup subcortical volume comparisons were also performed to explore the presence of atrophy in these structures (Supplementary Table 4). All regions except for the left thalamus showed significant effects, surviving FDR correction. The volume of these structures was significantly reduced in MSA patients compared with $\mathrm{HC}$ and PD patients, except for the amygdala, where no difference was found between MSA and PD patients. Significant volumetric reductions were observed in PD compared with $\mathrm{HC}$ in the bilateral nucleus accumbens and the left amygdala. No significant volumetric reductions were detected in HC compared with either patient group.

Classification. As a leave-one-out cross-validation (LOOCV) scheme was used to avoid overfitting by not using the test subject in the selection of features, we calculated a set of significant features at each iteration to pass them into the classification algorithm. Intergroup comparison followed by recurrent feature selection were 


\begin{tabular}{|l|l|l|l|l|}
\hline & HC $(\mathbf{n}=\mathbf{5 4})$ & PD $(\mathbf{n}=\mathbf{6 5})$ & MSA $(\mathbf{n}=\mathbf{3 1})$ & Stat $/ \mathbf{p}$ \\
\hline Left Amygdala & $0.2585(0.019)$ & $0.2535(0.020)$ & $0.2689(0.029)$ & $\mathrm{F}=5.6173 / \mathrm{p}=0.0144^{* 2,3}$ \\
\hline Left Ventral DC & $0.4327(0.024)$ & $0.4279(0.029)$ & $0.4116(0.027)$ & $\mathrm{F}=7.1122 / \mathrm{p}=0.004^{* 2,3}$ \\
\hline Right Thalamus & $0.4218(0.024)$ & $0.4155(0.025)$ & $0.3997(0.026)$ & $\mathrm{F}=9.7117 / \mathrm{p}=0.0018^{* 1-3}$ \\
\hline Right Ventral DC & $0.4731(0.033)$ & $0.4766(0.037)$ & $0.4462(0.030)$ & $\mathrm{F}=9.1112 / \mathrm{p}=0.0024^{* 2,3}$ \\
\hline Right Cerebellum & $0.3351(0.026)$ & $0.3357(0.020)$ & $0.2884(0.041)$ & $\mathrm{F}=37.0711 / \mathrm{p}=0.0018^{* 2,3}$ \\
\hline
\end{tabular}

Table 3. Significant Fractional Anisotropy (FA) of subcortical ROIs by group. HC: healthy controls; PD: Parkinson's disease patient group; MSA: multiple system atrophy patient group; * significant FDR corrected results; Post-hoc differences between $\mathrm{HC}$ and $\mathrm{PD}^{1}$; $\mathrm{HC}_{\text {and }} \mathrm{MSA}^{2}$; $\mathrm{PD}$ and $\mathrm{MSA}^{3}$; Stats refers to F-test (F).

\begin{tabular}{|l|l|l|l|l|}
\hline & HC $(\mathbf{n}=\mathbf{5 4})$ & PD $(\mathbf{n}=\mathbf{6 5})$ & MSA $(\mathbf{n}=\mathbf{3 1})$ & Stat/p \\
\hline Left Accumbens & $8.755 \cdot 10^{-4}\left(5.5 \cdot 10^{-5}\right)$ & $9.095 \cdot 10^{-4}\left(6.7 \cdot 10^{-5}\right)$ & $9.213 \cdot 10^{-4}\left(7.7 \cdot 10^{-5}\right)$ & $\mathrm{F}=4.5752 / \mathrm{p}=0.0354^{* 1,2}$ \\
\hline Left Putamen & $7.739 \cdot 10^{-4}\left(4.5 \cdot 10^{-5}\right)$ & $7.914 \cdot 10^{-4}\left(4.9 \cdot 10^{-5}\right)$ & $8.161 \cdot 10^{-4}\left(7.8 \cdot 10^{-5}\right)$ & $\mathrm{F}=6.9336 / \mathrm{p}=0.009^{* 1-3}$ \\
\hline Left Ventral DC & $1.206 \cdot 10^{-3}\left(9.9 \cdot 10^{-5}\right)$ & $1.232 \cdot 10^{-3}\left(1.1 \cdot 10^{-4}\right)$ & $1.313 \cdot 10^{-3}\left(1 \cdot 10^{-4}\right)$ & $\mathrm{F}=10.3436 / \mathrm{p}=0.0012^{* 2,3}$ \\
\hline Right Pallidum & $7.725 \cdot 10^{-4}\left(4.3 \cdot 10^{-5}\right)$ & $7.794 \cdot 10^{-4}\left(4.5 \cdot 10^{-5}\right)$ & $8.084 \cdot 10^{-4}\left(7.9 \cdot 10^{-5}\right)$ & $\mathrm{F}=5.2247 / \mathrm{p}=0.0212^{* 2,3}$ \\
\hline Right Thalamus & $1.24810^{-3}\left(1 \cdot 10^{-4}\right)$ & $1.271 \cdot 10^{-3}\left(1.1 \cdot 10^{-4}\right)$ & $1.350 \cdot 10^{-3}\left(1.1 \cdot 10^{-4}\right)$ & $\mathrm{F}=9.2948 / \mathrm{p}=0.0012^{* 2,3}$ \\
\hline Right Cerebellum & $9.187 \cdot 10^{-4}\left(7.5 \cdot 10^{-5}\right)$ & $9.162 \cdot 10^{-4}\left(7.1 \cdot 10^{-5}\right)$ & $1.141 \cdot 10^{-3}\left(1.9 \cdot 10^{-4}\right)$ & $\mathrm{F}=55.287 / \mathrm{p}=0.0012^{* 2,3}$ \\
\hline
\end{tabular}

Table 4. Significant Mean Diffusivity (MD) of subcortical ROIs by group. HC: healthy controls; PD: Parkinson's disease patient group; MSA: multiple system atrophy patient group; $*$ significant FDR corrected results; Posthoc differences between $\mathrm{HC}$ and $\mathrm{PD}^{1}$; $\mathrm{HC}$ and $\mathrm{MSA}^{2}$; $\mathrm{PD}$ and $\mathrm{MSA}^{3}$; Stats refers to F-test $(\mathrm{F})$.

employed to select the informative features to be introduced into the classifier. All 10 subcortical connections that showed significant group effects when using the whole sample were obtained in more than $80 \%$ of the iterations. Therefore, these connections seem to be stable in the feature selection procedure. The SVM classification algorithm correctly predicted overall group membership with an accuracy of 0.78 , with a sensitivity of 0.71 , and a specificity of 0.86 .

Considering the imbalanced group sizes and the differences in gender distribution in the overall sample, we repeated the entire procedure (feature selection and classification) using a matched subsample of PD patients and MSA patients, achieving an overall accuracy, sensitivity, and specificity of $0.84,0.77$, and 0.90 , respectively.

Additionally, we also evaluated the classification performance between MSA patients and HC. The SVM classifier achieved an overall accuracy, sensitivity, and specificity of $0.79,0.84$, and 0.74 , respectively.

Given that previous studies assessed the classification ability of other features extracted from subcortical structures such as diffusion tensor-derived metrics (e.g., FA and MD) and structure volume, we evaluated the performance of these metrics as well. A summary of these results is shown in Table 5. For these metrics, the previously described feature selection and classification procedure was employed. Subcortical FA and MD measures performed worse than NOS, concretely displaying a lower sensitivity. On the other hand, volumetric information from subcortical ROIs achieved a higher classification performance in all comparisons. Additionally, we evaluated whether combining multimodal features, in this case NOS and volumetric information, would improve the discrimination of MSA patients. We observed that introducing both sets of measures improved the sensitivity when differentiating MSA from HC.

\section{Discussion}

In this work, using a probabilistic connectivity approach, we have found evidence of reduced structural connectivity between subcortical structures in MSA patients compared with HC and with PD patients. We have also achieved a high accuracy in discriminating between PD and MSA patients using subcortical edge-wise tractography data and supervised machine learning.

We observed significant connectivity alterations in MSA patients, in agreement with previous findings and with our a priori hypothesis. Decreased NOS in MSA patients compared with both PD patients and HC was found in connections linked to basal ganglia such as the pallidum and putamen, the ventral diencephalon, the thalamus, and the cerebellum white matter/MCP.

As complementary analyses, we assessed diffusion tensor-derived metrics (FA and MD) and volumes of the 18 subcortical ROIs. In agreement with our structural connectivity results and previous studies ${ }^{30-34}$, we found abnormal diffusion tensor-derived measures and atrophy in the putamen, the pallidum, the ventral diencephalon, the thalamus, and the cerebellum. Concretely, we identified the cerebellum as the most damaged structure in terms of volumetric atrophy and abnormal diffusion measures, which agrees with the connectivity results as the connection between left and right cerebellum shows the most significant reduction of number of streamlines (NOS) between MSA patients and the other study groups. The vulnerability of the cerebellum in this disease is well-known and has been extensively described in previous studies ${ }^{30,35-37}$. Olivopontocerebellar atrophy is a hallmark of MSA, resulting in the characteristic ataxia. Abnormal basal ganglia volume, mostly in the putamen, is another common MSA feature ${ }^{36,38-41}$, as striatonigral degeneration is also detected at postmortem examination 


\begin{tabular}{|l|l|l|l|l|}
\hline & NOS & DT-metrics & SC vol & NOS + SC-vol \\
\hline MSA vs PD \\
\hline Accuracy & 0.79 & 0.75 & 0.84 & 0.84 \\
\hline Sensitivity & 0.71 & 0.58 & 0.74 & 0.74 \\
\hline Specificity & 0.86 & 0.92 & 0.94 & 0.94 \\
\hline MSA vs HC \\
\hline Accuracy & 0.79 & 0.72 & 0.85 & 0.89 \\
\hline Sensitivity & 0.84 & 0.61 & 0.77 & 0.84 \\
\hline Specificity & 0.74 & 0.82 & 0.93 & 0.94 \\
\hline MSA vs PD subsample \\
\hline Accuracy & 0.84 & 0.76 & 0.90 & 0.87 \\
\hline Sensitivity & 0.77 & 0.68 & 0.84 & 0.81 \\
\hline Specificity & 0.90 & 0.84 & 0.97 & 0.94 \\
\hline
\end{tabular}

Table 5. Discrimination performance of diffusion and structural features by groups. HC: healthy controls; PD: Parkinson's disease patient group; MSA: multiple system atrophy patient group; NOS: number of streamlines, DT-metrics: diffusion tensor-derived metrics, SC vol: subcortical volume.

in MSA patients. Thus, our findings of reduced NOS and atrophy involving the cerebellum and striationigral structures are in agreement with the previously described neuropathology of MSA.

While conventional DTI approaches, such as voxel-wise comparisons of FA and MD maps, have been previously used in characterizing MSA and distinguishing it from other diseases, limited work has been done in terms of assessing structural connectivity of MSA patients derived from tractography ${ }^{20,21,42,43}$. Thus, as our findings cannot be directly compared with previous published work, more studies should be performed to assess the generalizability of these results.

Regarding the PD group, no connectivity reductions were observed when compared with MSA patients, in agreement with previous studies ${ }^{6,30,44-46}$. Compared with HC, on the other hand, PD patients showed weakened connections between the putamen and the thalamus in the right hemisphere, as also previously described ${ }^{10,47-49}$. Connectivity results in PD agree with diffusion tensor-derived measures, as we found reduced FA in the putamen and thalamus in PD patients compared with HC, which is an indicator of microstructural disruption. Although previous studies have also reported subcortical atrophy of putamen and nucleus accumbens in $\mathrm{PD}^{36,50,51}$, in our sample, we were only able to detect volume reduction in the nucleus accumbens. Moreover, the strongest NOS reduction in MSA compared with PD was also the inter-cerebellar connection. Although cerebellar abnormalities have been reported in $\mathrm{PD}^{52}$, the cerebellum is not a key structure in the degenerative process of this disease. In this study, PD participants exhibit no detectable abnormalities in this structure. Concretely, PD patients did not differ from $\mathrm{HC}$ in inter-cerebellar connections, diffusion-tensor derived metrics nor cerebellar volume.

Considering these findings, it is difficult to speculate whether gray matter damage is the primary feature, subsequently leading to loss of white matter structural connectivity between subcortical regions, or whether the degenerative process of the MSA disease directly involves fiber abnormalities. Complementarily, the 10 connections found significantly reduced in MSA were correlated with subcortical volumes, to assess whether connectivity results were derived by the volumetric reduction. Only the NOS between the putamen and pallidum in the left hemisphere correlated with subcortical volumes $(\mathrm{r}=0.34, \mathrm{p}=0.031)$. Therefore, although ROIs size might have an effect in NOS, the connectivity results do not seem to be derived exclusively by this factor.

Given that MSA patients can be incorrectly diagnosed as having PD, especially in early stages of the disease, the development of neuroimaging biomarkers that can distinguish between these two illnesses with a high accuracy at the individual patient level would be of great clinical interest. Based on the vulnerability of certain subcortical structures since early phases of the MSA degenerative process, we hypothesized that the number of streamlines between these regions might have the ability to distinguish MSA from PD patients with a high accuracy. We found that the streamline count in the affected connections detected using edge-wise connectivity analysis was informative enough to distinguish MSA patients from PD patients with satisfactory accuracy, concretely of 0.78 and 0.84 , when using the whole dataset and a paired subsample, respectively. The selection of the brain connections used in this classification procedure was done through a combination of edge-wise intergroup comparisons and a recurrent feature elimination method. To avoid circularity, this step was nested in the LOOCV procedure, thereby never including the test subject in the comparison that defined the features used to train the classifier. Additionally, discrimination between MSA and HC shown accurate performance as well, concretely an overall accuracy of 0.79 .

Given that NOS measures showed a satisfactory classification performance, we qualitatively evaluated whether these features derived from tractography outperformed the diffusion tensor-derived metrics employed in previous studies ${ }^{24,25,32}$. For this reason, we complementarily assessed the discriminant ability of subcortical FA and MD measures. In our sample, diffusion tensor-derived metrics showed worse discrimination performance compared with the NOS features. These results suggest that tractography might provide more discriminant information than FA and MD. Additionally, we also assessed the classification performance of subcortical volumes, as previous studies also described their potential discriminant ability. The overall results showed that volume features outperformed the discrimination obtained through NOS analysis. However, it is noteworthy that the combination of volumetric and NOS features resulted in a better discrimination between MSA and HC. These results are in 
agreement with previous work highlighting the classification improvement observed when combining multimodal MRI data ${ }^{25}$.

Taken together, our results demonstrate the usefulness of NOS for correctly distinguishing MSA from PD and $\mathrm{HC}$, which outperformed the classification obtained with diffusion tensor-derived metrics. Although volumetric information was shown to be slightly superior to tractography measures, the combination of both sets of features improved the discrimination ability of the classifier.

The discriminating power of the connectivity features described in this study must also be demonstrated in future studies using samples of early-stage patients. Since abnormalities in subcortical structures occur early in MSA, this approach may prove to be appropriate in this setting. However, it is important to highlight that, although having shown promising results, for the moment, tractography methods are still not ready to be integrated in clinical routine. These techniques are very time-consuming and require visual inspection to ensure the quality of some steps, as well as technical knowledge to interpret the desired measurements. Therefore, more user-friendly platforms are required to integrate this methodology as part of a clinical diagnostic procedure.

Some limitations to the present study should be further addressed. First, given the relatively small sample size of MSA patient, we cannot assume that the set of discriminating network connections found in this study would be identified in other datasets; consequently, a larger sample is required to further validate these results. Complementary, a larger dataset would have allowed the use of k-fold cross-validation, a technique with smaller variance compared to the current leave-one-out cross-validation ${ }^{53}$.

Thirdly, in this study we performed a qualitative evaluation of multimodal models; thus, we cannot conclude whether the combination of different MRI features is an optimal approach to distinguish MSA from PD patients. In this context, further work should be carried out to find the most optimal combination of features and to quantitatively study the performance of this specific combination of multimodal data.

Fourthly, using subcortical gray matter structures as seeds is still challenging for diffusion tractography, as the tissue has an influence in the construction of the network, i.e.- using WM ROIs as seeds generates stronger networks with a larger number of connections than seeding in gray matter ROIs ${ }^{54}$. Finally, the characteristics of the reconstructed tracts are widely affected by the tractography techniques and parcellations employed. However, reduced integrity of subcortical connections in MSA patients has been extensively reported in previous MSA studies, which supports the validity of our findings.

\section{Conclusion}

In this work, we found evidence that WM structural connectivity between the cerebellum and the basal ganglia is weakened in MSA patients compared with controls and PD patients. Our results also suggest that these measures of connectivity may allow discriminating between MSA and PD patients at the individual patient level with a high accuracy, indicating a potential usefulness of this approach as a tool for differential diagnosis.

\section{Methods}

Participants. Thirty-eight MSA patients and sixty-seven PD patients were recruited from the Parkinson's Disease and Movement Disorders Unit, Hospital Clínic de Barcelona. Fifty-six healthy controls were recruited from the Institut de l'Envelliment, Universitat Autònoma de Barcelona and from friends or patients' spouses who volunteered to participate in the study. Detailed information of the sample can be found in our previous work ${ }^{55}$.

The inclusion criteria for patients were: (i) the fulfillment of the UK PD Society Brain Bank diagnostic criteria for PD and (ii) the fulfillment of the Gilman criteria ${ }^{56}$ for MSA.

Exclusion criteria consisted of: (i) MRI movement artifacts, (ii) pathological MRI findings other than mild WM hyperintensities or suggestive of alternative diagnoses in patients, (iii) significant neurological, systemic, or psychiatric comorbidity in the HC and PD groups, (iv) Mini-Mental State Examination scores $<25$ or dementia according to Movement Disorder Society criteria.

Two PD patients, six MSA patients, and one HC were excluded for excessive movement. One MSA patient was excluded for MR artifacts. One HC was excluded for WM hyperintensities. The final sample therefore consisted of $54 \mathrm{HC}, 31 \mathrm{MSA}$ patients, and $65 \mathrm{PD}$ patients.

Motor disease severity was evaluated using the Unified Multiple System Atrophy Rating Scale (motor section) for MSA patients, and the Unified Parkinson's Disease Rating Scale (motor section) for PD patients.

Written informed consent was obtained from the participants after full explanation of the procedures involved. The study was approved by the Ethics Committee of the Hospital Clinic and the University of Barcelona (HCB/2015/0798 and IRB00003099, respectively) and it was performed in accordance with relevant regulations and guidelines.

Basic statistical analyses. As described in our previous work ${ }^{57}$, intergroup comparisons of demographic, clinical, and imaging variables were performed with the general linear model using in-house MATLAB scripts. Statistical significance was established through the Monte Carlo simulations with 10000 permutations. Two-tailed $\mathrm{p}$-values were calculated as the proportion of values in the null distribution more extreme than those observed in the actual model. FDR was then used to control for multiple comparisons. In all analyses, gender was included as a covariate of no interest. Spearman correlations between clinical and imaging variables were evaluated using SPSS-24 (2016; Armonk, NY: IBM Corp.).

MRI acquisition. MRI data were acquired with a $3 \mathrm{~T}$ Siemens scanner (MAGNETOM Trio). The scanning protocol included high-resolution 3-dimensional T1-weighted images acquired in the sagittal plane $(\mathrm{TR}=2300 \mathrm{~ms}, \mathrm{TE}=2.98 \mathrm{~ms}, \mathrm{TI}=900 \mathrm{~ms}, 240$ slices, $\mathrm{FOV}=256 \mathrm{~mm} ; 1 \mathrm{~mm}$ isotropic voxel $)$, an axial FLAIR sequence $(\mathrm{TR}=9000 \mathrm{~ms}, \mathrm{TE}=96 \mathrm{~ms}$ ), and two sets of single band spin-echo diffusion-weighted MRI in the 


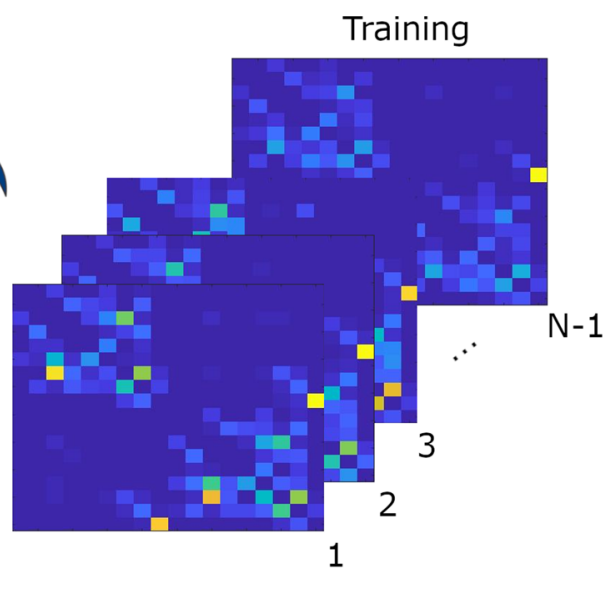

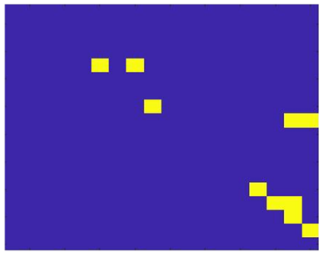

Significant connections

Multiply significant mask

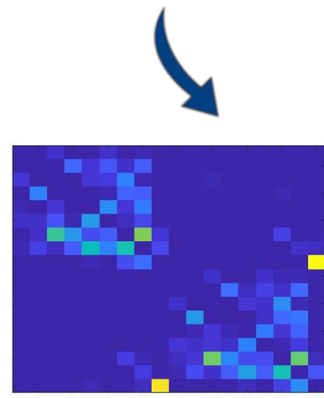

Test

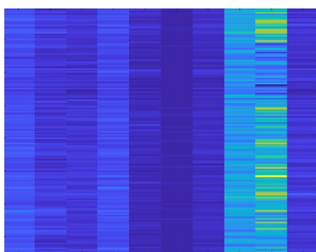

$\mathrm{N}-1$

Selected features used to train SVM

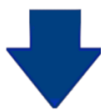

$\mathrm{RFE}+\mathrm{SVM}$

K-fold loop to tune SVM parameters

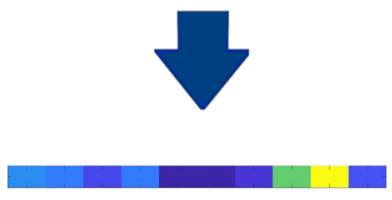

Test features

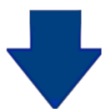

Predicted group

Figure 4. Classification procedure. Representation of one iteration of the feature selection and machine learning procedure. For each iteration, one subject was defined as the test set, whereas the remaining N-1 subjects made up the training set. Each training set was then fed into the TFNBS to calculate the significant connections between groups. Subsequently, the significant connections were introduced into a recursive feature elimination (RFE) algorithm to select the optimal connections. The support vector machine (SVM) algorithm was then tuned with the selected features. The resulting classifier model was then used to classify the corresponding test subject.

axial plane with opposite phase-encoding directions (anterior-posterior (AP) and posterior-anterior (PA)) $(\mathrm{TR}=7700 \mathrm{~ms}, \mathrm{TE}=89 \mathrm{~ms}, \mathrm{FOV}=244 \mathrm{~mm} ; 2 \mathrm{~mm}$ isotropic voxel). Diffusion-weighted images were acquired along 30 directions with a $b$ value $=1000 \mathrm{~s} / \mathrm{mm}^{2}$. An image without diffusion weighting $\left(b=0 \mathrm{~s} / \mathrm{mm}^{2}\right)$ was also acquired. Both sets of images were acquired at both AP and PA phase-encoding directions.

MRI preprocessing. Structural MRI preprocessing was performed using the automated FreeSurfer pipeline (version 5.1; https://surfer.nmr.mgh.harvard.edu/), which includes several independent steps: removal of non-brain tissue, automated Talairach transformation, intensity normalization ${ }^{58}$, tessellation of the gray matter/ WM boundary, automated topology correction ${ }^{59}$, and surface deformation to optimally place the gray matter/ 
$\mathrm{WM}$ and gray matter/cerebrospinal fluid boundaries ${ }^{27}$. The output of each step was visually inspected to guarantee correct and accurate preprocessing.

Manual inspection was initially performed to identify motion-related and intensity artifacts in the DWI images, which were subsequently preprocessed with FSL (version 5.08; https://fsl.fmrib.ox.ac.uk/fsl) using the FDT (FMRIB's Diffusion Toolbox) ${ }^{60}$. The DWI pipeline included several steps: brain extraction, susceptibility-induced distortion correction, and eddy-current distortion and subject motion correction. After the preprocessing, a diffusion tensor model was then fit at each voxel to the $4 \mathrm{D}$ DWI data series using DTIFit ${ }^{61}$. Fractional anisotropy (FA) and mean diffusivity (MD) maps were obtained as outputs of this process.

Regions of interest. Eighteen subcortical structures/regions (Fig. 1) were selected as regions of interest (ROIs) using the automated FreeSurfer segmentation ${ }^{26,27}$ : bilateral nucleus accumbens; amygdala; hippocampus; caudate nucleus; putamen; pallidum; thalamus; ventral diencephalon (including the hypothalamus, mammillary body, subthalamic nuclei, substantia nigra, red nucleus, lateral geniculate nucleus, and medial geniculate nucleus); and cerebellar white matter, including the middle cerebellar peduncles (MCP). These ROIs, generated in native structural space, were linearly registered to native diffusion space with FSL's Flirt in order to be used as seeds. The FA map from each subject was used as a reference image to compute the affine transformation from structural space to diffusion space using correlation ratio as a cost function. This transformation was then applied to the subcortical ROIs to transform them to native diffusion space. The resulting images were visually checked to assess the quality of the transformation. Each voxel was uniquely assigned to the mask with highest probability of membership to ensure that ROI masks did not overlap with each other due to resample blur.

DTI and volumetry data of subcortical structures (ROIs). Measures of mean FA and MD were calculated for each subcortical ROI to evaluate the microstructural diffusion properties of the 18 subcortical structures. Complementarily, the FreeSurfer volume-based stream ${ }^{62}$ was used to extract the volume (in $\mathrm{mm}^{3}$ ) of each ROI, which is often used as a marker of atrophy. Gender was included as a covariate in all analyses. Intracranial volume was also used as a covariate in the volumetric comparisons.

Brain network computation. The probability distribution of fiber directions in each voxel was calculated with Bedpost $\mathrm{x}^{63}$, which models diffusion signal as ball and stick components to generate a distribution of likely fiber orientations within each voxel ${ }^{61}$. Bedpostx runs Markov Chain Monte Carlo sampling to build up distributions on diffusion parameters at each voxel that can then be repeatedly sampled to allow voxel-to-voxel propagation of streamlines until stopping criteria are met $^{63}$. Probtrackx is used to compute the probabilistic connectivity between seeds ${ }^{63}$. It repeatedly samples from the principal diffusion direction probability distribution calculated in Bedpostx, at the voxel-wise level, creating a new streamline at each iteration ${ }^{64}$. For each seed region, 5000 streamlines were grown from each voxel with tracking parameters of $0.5 \mathrm{~mm}$ step size, 2000 maximum number of steps and $\mathrm{a} \pm 80^{\circ}$ curvature threshold. The strength of structural connectivity between each pair of regions was obtained from the number of reconstructed streamlines (NOS) between these ROIs. This process was repeated for all ROI pairs, to compute an $18 \times 18$ subcortical connectivity matrix. For each pair of ROIs, the total number of streamlines were considered: those starting from $i$ to $j$, and those initiated from $j$ to $i$, thus obtaining a symmetric structural connectivity matrix. As described in our previous work ${ }^{57}$, to reduce the risk of false-positive connections, streamlines intersecting fewer than two regions were ignored, and only connections between pairs of regions that were detected in at least $50 \%$ of subjects were considered ${ }^{65}$.

Characterization of structural connectivity differences. In order to test for intergroup differences in interregional NOS between PD, MSA, and HC, we used TFNBS ${ }^{28}$. This approach combines threshold-free cluster enhancement, a method frequently used in voxel-wise statistical inference ${ }^{66}$, and network-based statistics ${ }^{29}$, commonly used for statistical analysis of brain graphs. Initially, the symmetric $18 \times 18$ connectivity matrices $\mathrm{M}$ of all individual subjects underwent group testing, where an F statistic was calculated for each entry $i, j$ using the general linear model, producing a group $18 \times 18$ raw F statistic matrix Mstat. With TFNBS, the raw statistic value of each Mstat edge is replaced by its TFNBS score. Concretely, this score is determined by the heights of its neighboring edges (extension) and by the strength of the statistical effect (height); thus, the final TFNBS scores are influenced by how topologically "clustered" these effects are. Finally, a p value is ascribed to each entry in the TFNBS-enhanced matrix through permutation testing. Further description of this method can be found in Baggio et al. (2018).

Gender was included in the intergroup connectivity analyses as a covariate of no interest, and control of the false discovery rate (FDR) to $5 \%$ was used to correct for multiple testing across the connectome. Post-hoc comparisons were then tested in the significant connections found with TFNBS. Connectivity Fig. 3 was drawn using Surf Ice (www.nitrc.org).

Classification algorithm. LOOCV was implemented to avoid circularity in the classification (PD vs. MSA). When applying LOOCV, one subject is used for testing whereas the algorithm training is performed with the remaining N-1 subjects. This cross-validation technique enhances the generalization power of the classifier and prevents overfitting ${ }^{67}$.

In order to select the most important features, a mixed feature selection method was used for dimensionality reduction. Firstly, an intergroup comparison with TFNBS was computed to select an initial set of features. Secondly, a recursive feature elimination (RFE) method was employed to find the most optimal features to be introduced into the classifier (see Fig. 4). Finally, these features were entered into the classification algorithm to train the classifier. To test whether they were informative enough to differentiate PD from MSA patients, 
the trained algorithm was then applied to the test subject, with the whole process repeated $\mathrm{N}$ times. A detailed description of this method can be found in Abos et al. (2019).

The classification was assessed with a linear support vector machine (SVM), concretely using the SVM function from scikit-learn (http://scikit-learn.org), implemented in Python. Additionally, a C parameter needed to be defined, which determines the tradeoff between a correct classification of training examples and the maximization of the decision function's margin. In order to choose the best $\mathrm{C}$ parameter in an unbiased fashion, a cross-validated grid-search was employed.

The performance of the classification procedure was evaluated with the following measures: (i) accuracy (number of subjects correctly classified as PD or MSA patients divided by total number of subjects), (ii) sensitivity (number of MSA patients correctly classified divided by the total number of MSA patients), and (iii) specificity (number of PD patients correctly classified divided by the total number of PD patients). When assessing the ability of the classifier in discriminating between MSA patients and $\mathrm{HC}$, the specificity referred to the number of $\mathrm{HC}$ correctly classified divided by the total number of $\mathrm{HC}$.

\section{Data availability}

The data that support the findings of this study are available from the corresponding author upon reasonable request.

Received: 10 January 2019; Accepted: 2 October 2019;

Published online: 11 November 2019

\section{References}

1. Fanciulli, A. \& Wenning, G. K. Multiple-System Atrophy. N. Engl. J. Med. 372, 249-263 (2015).

2. Peeraully, T. \{Multiple\} \{System\} \{Atrophy\}. Semin. Neurol. 34, 174-181 (2014).

3. Baglieri, A. et al. Differences between conventional and nonconventional MRI techniques in Parkinson's disease. Funct. Neurol. 28, 73-82 (2013)

4. Savoiardo, M. Differential diagnosis of Parkinson's disease and atypical parkinsonian disorders by magnetic resonance imaging. Neurol. Sci. 24(Suppl 1), S35-7 (2003).

5. Wang, P. S., Wu, H. M., Lin, C. P. \& Soong, B. W. Use of diffusion tensor imaging to identify similarities and differences between cerebellar and Parkinsonism forms of multiple system atrophy. Neuroradiology 53, 471-481 (2011).

6. Tsukamoto, K. et al. Significance of apparent diffusion coefficient measurement for the differential diagnosis of multiple system atrophy, progressive supranuclear palsy, and Parkinson's disease: Evaluation by 3.0-T MR imaging. Neuroradiology 54, 947-955 (2012).

7. Lu, C. F. et al. Medullo-ponto-cerebellar white matter degeneration altered brain network organization and cortical morphology in multiple system atrophy. Brain Struct. Funct. 219, 947-958 (2014).

8. Shao, N., Yang, J. \& Shang, H. Voxelwise meta-analysis of gray matter anomalies in Parkinson variant of multiple system atrophy and Parkinson's disease using anatomic likelihood estimation. Neurosci. Lett. 587, 79-86 (2015).

9. Jeurissen, B., Descoteaux, M., Mori, S. \& Leemans, A. Diffusion MRI fiber tractography of the brain. NMR Biomed. e3785, https:// doi.org/10.1002/nbm.3785 (2017).

10. Menke, R. A. L. et al. Comprehensive morphometry of subcortical grey matter structures in early-stage Parkinson's disease. Hum. Brain Mapp. 35, 1681-1690 (2014).

11. Zhang, G. et al. Diffusion Kurtosis Imaging of Substantia Nigra is a Sensitive Method for Early Diagnosis and Disease Evaluation in Parkinson's Disease. Parkinsons. Dis. 2015, 1-5 (2015).

12. Ziegler, E. et al. Mapping track density changes in nigrostriatal and extranigral pathways in Parkinson's disease. Neuroimage 99, 498-508 (2014).

13. Son, S.-J., Kim, M. \& Park, H. Imaging analysis of Parkinson's disease patients using SPECT and tractography. Sci. Rep. 6, 38070 (2016).

14. Barbagallo, G. et al. Structural connectivity differences in motor network between tremor-dominant and nontremor Parkinson's disease. Hum. Brain Mapp. 38, 4716-4729 (2017).

15. Canu, E. et al. Brain structural and functional connectivity in Parkinson's disease with freezing of gait. Hum. Brain Mapp. 36, 5064-5078 (2015).

16. Fling, B. W. et al. Functional Reorganization of the Locomotor Network in Parkinson Patients with Freezing of Gait. PLoS One 9, e100291 (2014)

17. Wang, M. et al. Alterations of functional and structural connectivity of freezing of gait in Parkinson's disease. J. Neurol. 263, 1583-1592 (2016).

18. Galantucci, S. et al. Structural Brain Connectome and Cognitive Impairment in Parkinson Disease. Radiology 283, 515-525 (2017).

19. Vervoort, G. et al. Structural Brain Alterations in Motor Subtypes of Parkinson's Disease: Evidence from Probabilistic Tractography and Shape Analysis. PLoS One 11, e0157743 (2016).

20. Surova, Y. et al. Assessment of Global and Regional Diffusion Changes along White Matter Tracts in Parkinsonian Disorders by MR Tractography. PLoS One 8, (2013).

21. Surova, Y. et al. Disease-specific structural changes in thalamus and dentatorubrothalamic tract in progressive supranuclear palsy. Neuroradiology 57, 1079-1091 (2015).

22. Ito, M. et al. Usefulness of combined fractional anisotropy and apparent diffusion coefficient values for detection of involvement in multiple system atrophy. J. Neurol. Neurosurg. Psychiatry 78, 722-728 (2007).

23. Prodoehl, J. et al. Diffusion tensor imaging of Parkinson's disease, atypical parkinsonism, and essential tremor. Mov. Disord. 28, 1816-1822 (2013).

24. Du, G. et al. Combined Diffusion Tensor Imaging and Apparent Transverse Relaxation Rate Differentiate Parkinson Disease and Atypical Parkinsonism. AJNR. Am. J. Neuroradiol. 38, 966-972 (2017).

25. Péran, P. et al. MRI supervised and unsupervised classification of Parkinson's disease and multiple system atrophy. Mov. Disord. 33, 600-608 (2018)

26. Filipek, P. A., Richelme, C., Kennedy, D. N. \& Caviness, V. S. The young adult human brain: an MRI-based morphometric analysis. 4, 344-60 (1994).

27. Fischl, B. \& Dale, A. M. Measuring the thickness of the human cerebral cortex from magnetic resonance images. Proc. Natl. Acad. Sci. 97, 11050-11055 (2000).

28. Baggio, H. C. et al. Statistical inference in brain graphs using threshold-free network-based statistics. Hum. Brain Mapp. 39 (2018).

29. Zalesky, A., Fornito, A. \& Bullmore, E. T. Network-based statistic: Identifying differences in brain networks. Neuroimage 53, 1197-1207 (2010). 
30. Nicoletti, G. et al. Apparent diffusion coefficient measurements of the middle cerebellar peduncle differentiate the Parkinson variant of MSA from Parkinson's disease and progressive supranuclear palsy. Brain 129, 2679-2687 (2006).

31. Prakash, N. et al. Patterns of fractional anisotropy changes in white matter of cerebellar peduncles distinguish spinocerebellar ataxia-1 from multiple system atrophy and other ataxia syndromes. Neuroimage 47, T72-T81 (2009).

32. Nair, S. R. et al. A decision tree for differentiating multiple system atrophy from Parkinson's disease using 3-T MR imaging. Eur. Radiol. 23, 1459-1466 (2013).

33. Ji, L., Wang, Y., Zhu, D., Liu, W. \& Shi, J. White matter differences between multiple system atrophy (parkinsonian type) and parkinson's disease: A diffusion tensor image study. Neuroscience 305, 109-116 (2015).

34. Zanigni, S. et al. Accuracy of MR markers for differentiating Progressive Supranuclear Palsy from Parkinson's disease. NeuroImage Clin. 11, 736-742 (2016).

35. Dash, S. K. et al. Abnormalities of white and grey matter in early multiple system atrophy: comparison of parkinsonian and cerebellar variants. Eur. Radiol. 29, 716-724 (2019).

36. Heim, B., Krismer, F., De Marzi, R. \& Seppi, K. Magnetic resonance imaging for the diagnosis of Parkinson's disease. J. Neural Transm. 124, 915-964 (2017).

37. Zanigni, S. et al. White matter and cortical changes in atypical parkinsonisms: A multimodal quantitative MR study. Parkinsonism Relat. Disord. 39, 44-51 (2017).

38. Lee, J.-H. et al. Quantitative assessment of subcortical atrophy and iron content in progressive supranuclear palsy and parkinsonian variant of multiple system atrophy. J. Neurol. 260, 2094-2101 (2013).

39. Baudrexel, S. et al. The value of putaminal diffusion imaging versus 18-fluorodeoxyglucose positron emission tomography for the differential diagnosis of the Parkinson variant of multiple system atrophy. Mov. Disord. 29, 380-387 (2014).

40. Huppertz, H.-J. et al. Differentiation of neurodegenerative parkinsonian syndromes by volumetric magnetic resonance imaging analysis and support vector machine classification. Mov. Disord. 31, 1506-1517 (2016).

41. Scherfler, C. et al. Diagnostic potential of automated subcortical volume segmentation in atypical parkinsonism. Neurology 86, $1242-1249$ (2016).

42. Nilsson, C. et al. Tracking the neurodegeneration of parkinsonian disorders - a pilot study. Neuroradiology 49, 111-119 (2007).

43. Rosskopf, J. et al. Frontal Corpus Callosum Alterations in Progressive Supranuclear Palsy but Not in Parkinson's Disease. Neurodegener. Dis. 14, 184-193 (2014).

44. Rizzo, G. et al. Brain MR Contribution to the Differential Diagnosis of Parkinsonian Syndromes: An Update. Parkinsons. Dis. 2016 (2016).

45. Hattori, T. et al. Cognitive status correlates with white matter alteration in Parkinson's disease. Hum. Brain Mapp. 33, 727-739 (2012).

46. Worker, A. et al. Diffusion tensor imaging of Parkinson's disease, multiple system atrophy and progressive supranuclear palsy: A tract-based spatial statistics study. PLoS One 9 (2014).

47. Vaillancourt, D. E. et al. High-resolution diffusion tensor imaging in the substantia nigra of de novo Parkinson disease. Neurology 72, 1378-1384 (2009).

48. Péran, P. et al. Magnetic resonance imaging markers of Parkinson's disease nigrostriatal signature. Brain 133, 3423-3433 (2010).

49. Nagae, L. M. et al. Microstructural Changes within the Basal Ganglia Differ between Parkinson Disease Subtypes. Front. Neuroanat. 10, 17 (2016)

50. Nemmi, F., Sabatini, U., Rascol, O. \& Péran, P. Parkinson's disease and local atrophy in subcortical nuclei: insight from shape analysis. Neurobiol. Aging 36, 424-433 (2015).

51. Sterling, N. W. et al. Striatal shape in Parkinson's disease. Neurobiol. Aging 34, 2510-2516 (2013).

52. Wu, T. \& Hallett, M. The cerebellum in Parkinson's disease. Brain 136, 696-709 (2013).

53. Abós, A. et al. Discriminating cognitive status in Parkinson’s disease through functional connectomics and machine learning. Sci. Rep. 7 (2017).

54. Zajac, L., Koo, B.-B., Bauer, C. M., Killiany, R. \& Behalf Of The Alzheimer's Disease Neuroimaging Initiative, B. of the A. D. N. Seed Location Impacts Whole-Brain Structural Network Comparisons between Healthy Elderly and Individuals with Alzheimer's Disease. Brain Sci. 7 (2017).

55. Baggio, H. C. et al. Cerebellar resting-state functional connectivity in Parkinson's disease and multiple system atrophy: Characterization of abnormalities and potential for differential diagnosis at the single-patient level. NeuroImage Clin. 22, 101720 (2019).

56. Gilman, S. et al. Second consensus statement on the diagnosis of multiple system atrophy. Neurology 71, 670-676 (2008).

57. Abos, A. et al. Disrupted structural connectivity of fronto-deep gray matter pathways in progressive supranuclear palsy. NeuroImage Clin. 23 (2019).

58. Sled, J. G., Zijdenbos, A. P. \& Evans, A. C. A nonparametric method for automatic correction of intensity nonuniformity in MRI data. IEEE Trans. Med. Imaging 17, 87-97 (1998).

59. Segonne, F., Pacheco, J. \& Fischl, B. Geometrically Accurate Topology-Correction of Cortical Surfaces Using Nonseparating Loops. IEEE Trans. Med. Imaging 26, 518-529 (2007).

60. Jbabdi, S., Sotiropoulos, S. N., Savio, A. M., Graña, M. \& Behrens, T. E. J. Model-based analysis of multishell diffusion MR data for tractography: How to get over fitting problems. Magn. Reson. Med. 68, 1846-1855 (2012).

61. Behrens, T. E. J. et al. Characterization and propagation of uncertainty in diffusion-weighted MR imaging. Magn. Reson. Med. 50, 1077-1088 (2003).

62. Fischl, B. et al. Whole brain segmentation: automated labeling of neuroanatomical structures in the human brain. Neuron 33, 341-55 (2002).

63. Behrens, T. E. J., Berg, H. J., Jbabdi, S., Rushworth, M. F. S. \& Woolrich, M. W. Probabilistic diffusion tractography with multiple fibre orientations: What can we gain? Neuroimage 34, 144-155 (2007).

64. Zhan, L. et al. Comparison of nine tractography algorithms for detecting abnormal structural brain networks in Alzheimer's disease. Front. Aging Neurosci. 7, 48 (2015).

65. Zalesky, A. et al. Connectome sensitivity or specificity: which is more important? Neuroimage 142, 407-420 (2016)

66. Smith, S. M. \& Nichols, T. E. Threshold-free cluster enhancement: Addressing problems of smoothing, threshold dependence and localisation in cluster inference. Neuroimage 44, 83-98 (2009).

67. Luntz, A. On estimation of characters obtained in statistical procedure of recognition. Tech. Kibern (1969).

\section{Acknowledgements}

We are most grateful to the neurologists involved in the Catalan multiple system atrophy-registry (CMSAR), the patients, their families and control subjects for their cooperation. We are also indebted to the Magnetic Resonance Imaging core facility of the IDIBAPS for the technical support; and we would also like to acknowledge the CERCA Programme/Generalitat de Catalunya. This study was sponsored by the Spanish Ministry of Economy and Competitiveness (PSI2013-41393-P; PSI2017-86930-P cofinanced by Agencia Estatal de Investigación (AEI) and the European Regional Development Fund), by Generalitat de Catalunya (2017SGR748) and by Fundació La 
Marató de TV3 in Spain (20142310; PI043296). A.A. was supported by a 2016 fellowship from the Departament d'Empresa i Coneixement de la Generalitat de Catalunya, AGAUR (2016FI_B 00360), A.C. was supported by APIF predoctoral fellowship from the University of Barcelona (2017-2018) and CU was supported by a fellowship from 2014, Spanish Ministry of Economy and Competitiveness (BES-2014-068173) and cofinanced by the European Social Fund (ESF). M.J.M. has received funding from Fondo de Investigaciones Sanitarias of Spain (FIS PI17/00096) and from Generalitat de Catalunya (AGAUR 2017SGR1502). A.A., H.C.B., B.S., A.C., C.U., D.M., A.P., E.M. and C.J. report no disclosures. M.J.M. received honoraria for advice and lecture from Abbvie, Bial and Merzt Pharma and grants from Michael J. Fox Foundation for Parkinson Disease (MJFF): MJF_PPMI_10_001, PI044024. Y.C. has received funding in the past 5 years from FIS/FEDER, H2020 programme Union Chimique Belge (UCB pharma), Teva, Medtronic, Abbvie, Novartis, Merz, Piramal Imaging, and Esteve, Bial and Zambon. Y.C. is currently associate editor of Parkisonism and Related Disorders. NITRC, NITRC-IR, and NITRC-CE have been funded in whole or in part with Federal funds from the Department of Health and Human Services, National Institute of Biomedical Imaging and Bioengineering, the National Institute of Neurological Disorders and Stroke, under the following NIH grants: 1R43NS074540, 2R44NS074540, and 1U24EB023398 and previously GSA Contract No. GS-00F-0034P, Order Number HHSN268200100090U.

\section{Author contributions}

A.A. took part in research project execution, study design, preprocessing, data analysis, and drafting of the manuscript; H.C.B. took part in research project design and execution, study design, and review and critique of the results; B.S. took part in research project design and execution, study design, and review and critique of the results; A.C. took part in research project execution, study design, data analysis; C.U. took part in research project execution and preprocessing. D.M.G., A.P.S. and E.M. took part in research project execution; Y.C., C.J. and M.J.M. took part in research project design and execution, study design, and review and critique of the results. All authors reviewed the manuscript.

\section{Competing interests}

The authors declare no competing interests.

\section{Additional information}

Supplementary information is available for this paper at https://doi.org/10.1038/s41598-019-52829-8.

Correspondence and requests for materials should be addressed to M.J.M.

Reprints and permissions information is available at www.nature.com/reprints.

Publisher's note Springer Nature remains neutral with regard to jurisdictional claims in published maps and institutional affiliations.

Open Access This article is licensed under a Creative Commons Attribution 4.0 International License, which permits use, sharing, adaptation, distribution and reproduction in any medium or format, as long as you give appropriate credit to the original author(s) and the source, provide a link to the Creative Commons license, and indicate if changes were made. The images or other third party material in this article are included in the article's Creative Commons license, unless indicated otherwise in a credit line to the material. If material is not included in the article's Creative Commons license and your intended use is not permitted by statutory regulation or exceeds the permitted use, you will need to obtain permission directly from the copyright holder. To view a copy of this license, visit http://creativecommons.org/licenses/by/4.0/.

(C) The Author(s) 2019 